\title{
Phylogenetic Characterization of a Novel Insect-Specific Flavivirus Detected in a Culex Pool, Collected from Assam, India
}

\author{
Sibnarayan Datta Reji Gopalakrishnan Soumya Chatterjee Vijay Veer \\ Defence Research Laboratory (DRDO), Tezpur, India
}

\section{Key Words}

Flavivirus - Reverse transcriptase PCR · Culex · Nonstructural protein 5 - Phylogenetic analysis - Northeastern India,

Assam

\begin{abstract}
Objective: We report the phylogenetic characterization of a unique flavivirus sequence detected in a wild Culex tritaeniorhynchus mosquito pool, collected from the northeast Indian state of Assam. Methods: DNA and RNA were extracted from field-collected mosquito pools. Extracts were subjected to PCR and reverse transcriptase PCR amplification using universal and type-specific primers for direct detection of flavivirus-specific viral nucleic acids. An amplified flavivirus nonstructural protein 5 (NS5) genetic region was sequenced and BLAST searched, and phylogenetic analyses performed with reference sequences retrieved from GenBank. Results: Phylogenetic analyses revealed the sequence to be related to insect-specific flaviviruses (ISFs) of the genus Flavivirus, family Flaviviridae. Despite being related to the Palm Creek virus (PCV; an ISF very recently reported from Northern Australia), the present sequence (provisionally named Assam virus) was found to be highly divergent from PCV and other ISF sequences available in GenBank. The partial NS5 sequence analysis demonstrated low nucleotide sequence identity
\end{abstract}

(66-77\%) with known ISFs reported from other parts of the globe. Conclusion: Findings of this study suggest the presence of a candidate novel ISF - the first to be reported from India.

(c) 2015 S. Karger AG, Basel

\section{Introduction}

Flavivirus is the most diverse genus of the Flaviviridae family, consisting of more than 70 known viruses, including human pathogens such as Japanese encephalitis virus, West Nile virus, dengue virus, yellow fever virus, tickborne encephalitis virus, and Kyasanur forest disease virus. Owing to their potential to cause high mortality, many of these flaviviruses pose serious global health risks and some are even enlisted as biothreat/priority agents [1].

The majority of the flaviviruses are transmitted by mosquitoes (mosquito-borne viruses) and ticks (tickborne viruses) [2]. However, another group (no known vector) of flaviviruses exists for whom vectors still remain unidentified [2]. Over time, an additional lineage called the insect-specific flaviviruses (ISFs) has been recognized, and these can infect and replicate in insect cells but not in vertebrate cells [3]. Cell-fusing agent virus is the arche- 
type virus of this group. Several other ISFs were also identified subsequently, such as the Kamiti river virus, Culex flavivirus (CxFV), Aedes flavivirus, Nakiwogo virus (NAKV), Quang Binh virus, Calbertado virus and Palm Creek virus (PCV) [2, 4-7].

ISFs, apart from providing important insights into flavivirus evolutionary dynamics, have also been suggested to modulate vector competence and transmission efficiency of human pathogenic flaviviruses $[3,4,7-11]$. Interestingly, possibly via superinfection exclusion, as previously described for other viruses [12-15], recent studies have provided evidence that prior infection of cell cultures with an ISF may lead to reduced permissiveness and replication efficiency of certain human pathogenic flaviviruses $[7,11]$. However, the clinical significance of ISFs has increased enormously since it was reported that the pathogenicity of certain human-infecting flaviviruses may actually be augmented by ISFs under specific conditions $[9,10]$. Consequently, a large number of research groups have focused their research objectives on ISF discovery and characterization, which has led to the identification of a number of ISFs in the last few years.

In this article we report our findings and phylogenetic characterization of a novel Culex-associated flavivirus sequence - the first to be detected and reported from India.

\section{Materials and Methods}

Sample Collection, Pooling and Nucleic Acid Purification

The sequence analyzed in this work was detected from a pool of Culex tritaeniorhynchus mosquitoes caught in the wild, collected from Tezpur, Assam, India (coordinates: $26.63^{\circ} \mathrm{N} 92.8^{\circ} \mathrm{E}$; elevation: $77 \mathrm{~m}$; mean temperature: $25.1^{\circ}$; mean relative humidity: $63 \%)$. Mosquito collection and virus detection is a part of an arbovirus surveillance program that is currently being pursued by our laboratory. Following field collection, mosquitoes were identified using standard keys $[16,17]$, sorted (according to species and gender) and sent to the laboratory in preservative (RNAlater; SigmaAldrich, USA). Twenty-six pools of mosquitoes (consisting of a total of 2,350 mosquitoes; $80-100$ mosquitoes per pool) belonging to 8 different species of the genera Culex and Mansonia (10 pools of $C$. quinquefasciatus; 3 pools each of $C$. tritaeniorhynchus, $C$. vishnui and M. uniformis; 2 pools each of C. gelidus, C. bitaeniorhynchus and $M$. annulifera; 1 pool of $C$. pseudovishnui) were processed for reverse transcriptase PCR (RT-PCR)-based molecular detection of arboviruses, belonging to the family Flaviviridae. For extraction of total nucleic acids, minipools were made $(\sim 20-25$ mosquitoes), ground in lysis buffer using sterile micropestles, followed by column purification and elution in $50 \mu$ l of nuclease-free water, according to the manufacturer's protocol (High Pure Viral Nucleic Acid Kit; Roche, Germany). Purified nucleic acid extracts were checked on conventional and denaturing agarose gel, and quantified using a microvolume UV-Vis spectrophotometer ( $\mathrm{Pi}$ - codrop, UK). An aliquot of nucleic acid was treated with amplification grade Deoxyribonuclease I (Sigma-Aldrich) for use as a template in an RT-PCR reaction. Another aliquot of the nucleic acid was treated with DNase free RNase A (Sigma-Aldrich) for use in PCR.

\section{Reverse Transcriptase PCR, PCR and Sequencing}

Single-tube RT-PCR was performed using an enhanced Avian HS RT-PCR kit according to the manufacturer's protocol (Sigma). RNA quality was verified by RT-PCR using primers Act-2F and Act-8R, as described elsewhere [18]. For detection of flaviviruses, generic primers CFDDJ9979 and FUDJ9166 were used as described previously $[19,20]$. These primers anneal to highly conserved regions of the nonstructural protein 5 (NS5) encoding region (producing a $\sim 830$-bp amplicon). In addition, RNase Atreated DNA extract was also subjected to PCR under the same reaction conditions, except for the initial reverse transcription step which was omitted (negative control). Amplified RT-PCR products were subsequently used in seminested PCR for detecting different human pathogenic flaviviruses, using primers described elsewhere $[19,20]$. Following gel electrophoresis, amplicons were sliced out and purified (Qiagen, India), and both strands were sequenced (Biolinkk, India). The sequence data were end-trimmed, checked, manually edited and matched using BioEdit 7.0.5.3 [21].

\section{Sequence BLAST Search and Phylogenetic Analysis}

Homologous nucleotide and translated amino acid sequences were searched in the NCBI nonredundant database using megablast/blastn and blastp programs, respectively, keeping search parameters at default values [22]. Nucleotide sequences accessions obtained in the BLAST search were retrieved from GenBank and aligned with the present sequence using the MUSCLE 3.7 program in full processing mode for maximum accuracy, while translated amino acid sequences were aligned using ClustalW incorporated in the BioEdit program $[2,3,21,23,24]$. Phylogenetic relatedness of the sequences was inferred using the neighbor-joining method. The evolutionary distances for nucleotides and amino acid sequences were computed using the maximum composite likelihood method and Poisson correction methods, respectively, and were expressed in the units of the number of base substitutions per site. The reliability of pairwise comparison and phylogenetic trees was evaluated by bootstrap resampling (1,000 replicates). Molecular evolutionary analyses were carried out using MEGA 5.2 [25].

\section{Results}

RT-PCR amplification of the RNA extract with flavivirus generic primers yielded an amplicon of $\sim 830 \mathrm{bp}$. However, seminested PCR for detection of 11 different human pathogenic flaviviruses, using the RT-PCR-generated amplicon as a template, did not yield any positive signal. The RT-PCR amplicon was sequenced and analyzed, and sequence data were submitted to GenBank (available under accession No. KF006336). No amplicon was detected in the PCR reaction tube containing DNA 


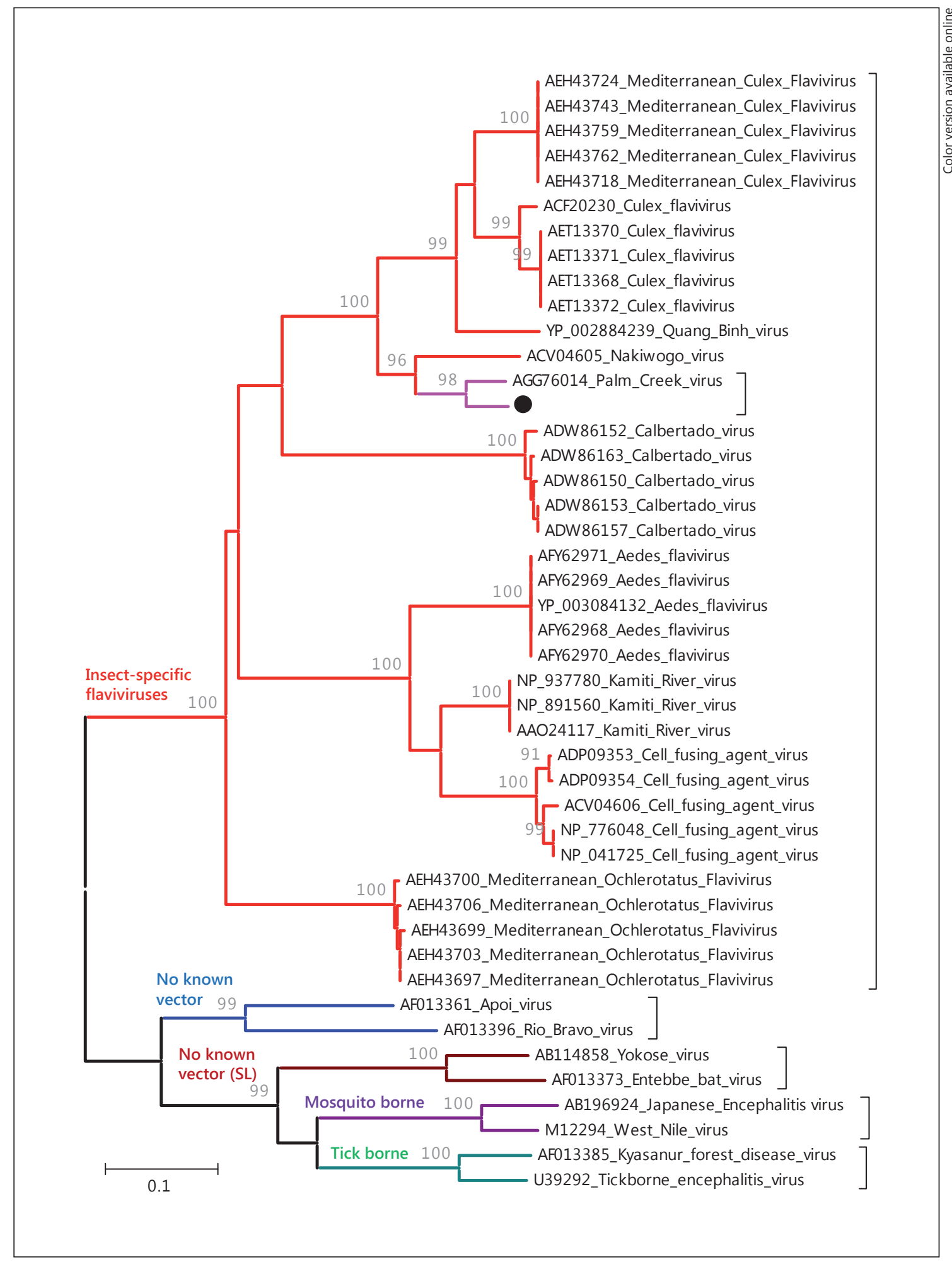

Fig. 1. Neighbor-joining tree showing phylogenetic relationships, based on similarity among 45 amino acid sequences is represented. The sequence obtained in the present study is indicated by the black solid circle (1). The tree is drawn to scale, with branch lengths in the same units as those of the evolutionary distances used to infer the phylogenetic tree. Gaps and missing data were eliminated and the final dataset contains a total of 189 positions. The percentage of replicate trees in which the associated taxa clustered together in the bootstrap test $(1,000$ replicates $)$ is shown at the branch nodes. 
extracts. The absence of the amplicon in this tube was interpreted as the absence of flavivirus sequences in DNA form or as integrated into the host genome.

Initially, the megablast search did not yield any match with sequences available in GenBank (nonredundant database). However, in the blastn search (for a somewhat similar sequence), the top 100 hits showed considerable matches with different ISF sequences, but not with any flavivirus of human clinical significance. The highest scoring match $(77 \%$ nucleotide identity and $92 \%$ amino acid identity) was observed with PCV (GenBank accession No. KC505248), which was recently reported from Northern Australia. The second highest match (73\% nucleotide identity and $85 \%$ amino acid identity) was documented with NAKV (GenBank accession No. GQ165809), which was isolated from Uganda, followed by a match with Mediterranean CxFVs (71\% nucleotide identity and 79\% amino acid identity; GenBank accession Nos. JF707852, JF707849, JF707827 and JF707815). This was followed by matches with sequences of Mediterranean $\mathrm{CxFV}$, Culex theileri flavivirus, Quang Binh virus and Calbertado virus, which were isolated and submitted from different parts of the globe.

In the next step, we searched for homologous sequences in the databases using translated amino acids sequence and the blastp algorithm, which detected a putative conserved domain of flavivirus NS5 protein within the query amino acid sequence (also confirmed in the NCBI conserved domain database). Similar to blastn results, blastp also reported the top 100 hits to be of flavivirus origin, but none of them were a clinically relevant flavivirus. In complete agreement with blastn, the highest match was observed with PCV polyprotein (GenBank accession No. AGG76014), followed by NAKV polyprotein (GenBank accession No. ACV04605) and further with different sequences from Mediterranean $\mathrm{CxFV}$, Culex theileri flavivirus, Quang Binh virus, Calbertado virus, Aedes flavivirus, etc. The blastp search of the present sequence also revealed amino acid identity with a number of sequences from the no-known-vector group, although with a lesser percentage.

To further confirm and correctly ascertain the phylogenetic relatedness of our query sequence to those of other flaviviruses, we performed a phylogenetic analysis of the nucleotide sequence as well as the amino acid sequence with other sequences retrieved from GenBank, as references. We found the branching patterns of our phylogenetic trees to be principally similar to previously reported phylogenies $[2,3]$. In phylogenetic analysis, our query sequence undoubtedly clustered within the Culex- associated flavivirus clade within the ISFs, showing the closest phylogenetic relatedness with a PCV sequence with high bootstrap values, supporting BLAST results (fig. 1). An alignment of the amino acid sequence (partial NS5 region) of the present ISF sequence, along with other GenBank reference sequences is provided as online supplementary data (for all online suppl. material, see www.karger.com/doi/10.1159/000381901).

\section{Discussion}

Based on the analysis of partial NS5 sequences, we illustrate that despite being phylogenetically related to the $\mathrm{CxFV}+$ Quang Binh virus + NAKV clade of ISFs [3], the present sequence is vastly divergent. It demonstrated only 77\% pairwise nucleotide sequence identity over the partial NS5 region with the phylogenetically closest virus $(\mathrm{PCV})$, which is far less than the recommended criterion of $>84 \%$ pairwise nucleotide sequence identity required to be classified as the same 'species' as PCV [2]. Thus, considering the above criteria and with reference to recent publications based on phylogenetic analysis of partial NS5 sequences to classify novel flaviviruses [6, 8, 2629], we provisionally named this sequence 'Assam virus' (named after the state from where it was first detected) to distinguish it from viruses reported from other parts of the world.

Although very unusual for flaviviruses, the integration of flavivirus sequences in the mosquito genome has recently been reported in some studies [26, 30,31], but not detected in other studies [28]. Our study also does not suggest such integration phenomena for the present virus. Recent evidence showing the potential of ISFs to coexist and modulate the transmission efficiency of certain human pathogenic flaviviruses has gained significant interest. However, the phenomenon remains to be further validated since some studies report competitive suppression of human pathogenic flaviviruses by ISFs [7, 11], while others report the enhancement of transmission efficiency of human pathogenic flaviviruses by $\operatorname{ISF}[9,10]$. Even though, the surveillance of known human pathogenic arboviruses has led to the discovery of several novel viruses including ISFs [3, 5-8, 10, 28, 29, 32], but their significance in transmission and pathogenesis remains to be elucidated.

Although virus isolation is considered as the gold standard for identification, it requires a cell culture facility with certain levels of a biosafety environment [33]. In contrast, RT-PCR is rapid, highly sensitive, specific and 
inexpensive as compared to virus isolation-based methods. Additionally, high throughput is possible with RTPCR for routine screening of a large number of fieldcaught mosquitoes and identification of viruses. In some previous studies, apart from the NS5 region, sequence analysis of other genetic regions such as envelope (E) and NS3 regions have been recommended. However, we selected the highly conserved enzymatic motifs of the NS5 sequence since the phylogenetic analysis in this region has been considered imperative for the characterization of new flaviviruses [31]. In continuation of our present findings, our efforts to sequence and analyze more genetic regions of this virus are in progress.

We acknowledge that there are indeed some limitations of the present study. Although phylogenetic analyses of the viral genetic sequences are indispensable, characterization of antigens and infectivity assays using mammalian/ insect cell lines are additionally required to establish the novelty of virus isolates. However, in this surveillance study, vectors were collected and preserved with the aim of directly detecting viral sequences through RT-PCR. Therefore, we speculate that our attempts to infect susceptible insect cell lines with this mosquito pool homogenate were futile possibly due to the antigen-denaturing effects of the preserving solution.

Though India has the well-organized National Vector Borne Disease Control Program (www.nvbdcp.gov.in/), which principally focuses on identification/control of vectors and treatment, regular surveillance for emerging/ reemerging arboviruses in mosquito vectors is not routine. Apart from reports on virus isolation studies [34], we could find only two reports from India that focused on systematic survey and detection of Japanese encephalitis virus in mosquito vectors $[35,36]$. We have thus undertaken an arbovirus surveillance program in the northeastern parts of India, where vector-borne viruses such as Japanese encephalitis virus, dengue virus, and chikungunya virus have been reported to be endemic or to have emerged recently [36-39].

In conclusion, a putatively novel ISF is reported for the first time from India that adds additional dimension to worldwide flavivirus distribution and diversity. Apart from arbovirus surveillance to monitor endemic/emerging human pathogenic flaviviruses in this part of India, further entomologic investigations to explore the ISF biological relevance to pathogenic flaviviruses are essential.

\section{Acknowledgements}

This study was supported by funds from the Defence Research and Development Organization (DRDO), Ministry of Defence, Government of India. We sincerely thank the anonymous reviewers for their useful suggestions and advice.

\section{References}

1 NIAID Category A, B, and C Priority Pathogens List. www.niaid.nih.gov/topics/biodefenserelated/biodefense/pages/cata.aspx (accessed May 1, 2013).

2 Kuno G, Chang GJ, Tsuchiya KR, Karabatsos N, Cropp CB: Phylogeny of the genus Flavivirus. J Virol 1998;72:73-83.

-3 Cook S, Moureau G, Kitchen A, Gould EA, de Lamballerie X, Holes EC, Harbach RE: Molecular evolution of the insect-specific flaviviruses. J Gen Virol 2012;93:223-234.

4 Crabtree MB, Nga PT, Miller BR: Isolation and characterization of a new mosquito flavivirus, Quang Binh virus, from Vietnam. Arch Virol 2009;154:857-860.

5 Junglen S, Kopp A, Kurth A, Pauli G, Ellerbrok H, Leendertz FH: A new flavivirus and a new vector: characterization of a novel flavivirus isolated from uranotaenia mosquitoes from a tropical rain forest. J Virol 2009;83: 4462-4468.
6 Bolling BG, Eisen L, Moore CG, Blair CD: Insect-specific flaviviruses from Culex mosquitoes in Colorado, with evidence of vertical transmission. Am J Trop Med Hyg 2011;85: 169-177.

$\checkmark 7$ Hobson-Peters J, Yam AW, Lu JW, Setoh YX, May FJ, Kurucz N, Walsh S, Prow NA, Davis SS, Weir R, Melville L, Hunt N, Webb RI, Blitvich BJ, Whelan P, Hall RA: A new insect-specific flavivirus from northern Australia suppresses replication of West Nile virus and Murray Valley encephalitis virus in co-infected mosquito cells. PLoS One 2013;8:e56534.

-8 Farfan-Ale JA, Lorono-Pino MA, Garcia-Rejon JE, Hovav E, Powers AM, Lin M, Dorman KS, Platt KB, Bartholomay LC, Soto V, Beaty BJ, Lanciotti RS, Blitvich BJ: Detection of RNA from a novel West Nile like virus and high prevalence of an insect-specific flavivirus in mosquitoes in the Yucatan Peninsula of Mexico. Am J Trop Med Hyg 2009;80:85-95.

$\checkmark 9$ Kent RJ, Crabtree MB, Miller BR: Transmission of West Nile virus by Culex quinquefasciatus say infected with Culex flavivirus Izabal. PLoS Negl Trop Dis 2010;4:e671.
10 Newman CM, Cerutti F, Anderson TK, Hamer GL, Walker ED, Kitron UD, Ruiz MO, Brawn JD, Goldberg TL: Culex flavivirus and West Nile virus mosquito coinfection and positive ecological association in Chicago, United States. Vector Borne Zoonotic Dis 2011;11:1099-1105.

11 Bolling BG, Olea-Popelka FJ, Eisen L, Moore CG, Blair CD: Transmission dynamics of an insect-specific flavivirus in a naturally infected Culex pipiens laboratory colony and effects of co-infection on vector competence for West Nile virus. Virology 2012;427:90-97.

12 Karpf AR, Lenches E, Strauss EG, Strauss JH, Brown DT: Superinfection exclusion of alphaviruses in three mosquito cell lines persistently infected with Sindbis virus. J Virol 1997;71:7119-7123.

13 Burivong P, Pattanakitsakul SN, Thongrungkiat S, Malasit P, Flegel TW: Markedly reduced severity of dengue virus infection in mosquito cell cultures persistently infected with Aedes albopictus densovirus (AalDNV). Virology 2004;329:261-269. 
14 Pepin KM, Lambeth K, Hanley KA: Asymmetric competitive suppression between strains of dengue virus. BMC Microbiol 2008; 8:28.

15 Pesko K, Mores CN: Effect of sequential exposure on infection and dissemination rates for West Nile and St. Louis encephalitis viruses in Culex quinquefasciatus. Vector Borne Zoonotic Dis 2009;9:281-286.

16 Barraud PJ: Diptera: family Culicidae, tribe Megarhini and Culicini; in: The Fauna of British India Including Ceylon and Burma. London, Taylor and Francis, 1934, vol 5.

17 Reuben R, Tewari SC, Hiriyan J, Akiyama J: Illustrated keys to species of Culex (Culex) associated with Japanese encephalitis in Southeast Asia (Diptera: Culicidae). Mosq Syst 1994;26:75-96.

18 Staley M, Dorman KS, Bartholomay LC, Fernández-Salas I, Farfan-Ale JA, Loroño-Pino MA, Garcia-Rejon JE, Ibarra-Juarez L, Blitvich BJ: Universal primers for the amplification and sequence analysis of actin- 1 from diverse mosquito species. J Am Mosq Control Assoc 2010;26:214-218.

19 Chang GJ, Trent DW, Vorndam AV, Vergne E, Kinney RM, Mitchell CJ: An integrated target sequence and signal amplification assay, reverse transcriptase-PCR-enzyme-linked immunosorbent assay, to detect and characterize flaviviruses. J Clin Microbiol 1994;32: 477-483.

20 Krauss H, Weber A, Appel M, Enders B, Isenberg HD, Schiefer HG, Slenczka W, von Graevenitz A, Zahner H: Zoonosis - Infectious Diseases Transmissible from Animals to $\mathrm{Hu}$ mans, ed 3. Washington, American Society for Microbiology, 2003, p 474.

21 Hall TA: BioEdit: a user-friendly biological sequence alignment editor and analysis program for windows 95/98/NT. Nucl Acids Symp Ser 1999;41:95-98.

22 Altschul SF, Madden TL, Schäffer AA, Zhang J, Zhang Z, Miller W, Lipman DJ: Gapped BLAST and PSI-BLAST: a new generation of protein database search programs. Nucleic Acids Res 1997;25:3389-3402.
23 Cook S, Holmes EC: A multigene analysis of the phylogenetic relationships among the flaviviruses (family: Flaviviridae) and the evolution of vector transmission. Arch Virol 2006; 151:309-325.

24 Edgar RC: MUSCLE: multiple sequence alignment with high accuracy and high throughput. Nucleic Acids Res 2004;32:17921797.

25 Tamura K, Dudley J, Nei M, Kumar S: MEGA4: Molecular Evolutionary Genetics Analysis (MEGA) software version 4.0. Mol Biol Evol 2007;24:1596-1599.

26 Roiz D, Vazquez A, Seco MP, Tenorio A, Rizzoli A: Detection of novel insect flavivirus sequences integrated in Aedes albopictus (Diptera: Culicidae) in Northern Italy. Virol J 2009;6:93.

27 Tyler S, Bolling BG, Blair CD, Brault AC, Pabbaraju K, Armijos V, Clark DC, Calisher CH, Drebot MA: Distribution and phylogenetic comparisons of a novel mosquito flavivirus sequence present in Culex tarsalis Mosquitoes from Western Canada with viruses isolated in California and Colorado. Am J Trop Med Hyg 2011;85:162-168.

28 Calzolari M, Zé-Zé L, Růžek D, Vázquez A, Jeffries C, Defilippo F, Costa Osorio H, Kilian P, Ruiz S, Fooks AR, Maioli G, Amaro F, Tlusty M, Figuerola J, Medlock JM, Bonilauri P, Alves MJ, Sebesta O, Tenorio A, Vaux AGC, BelliniR, Gelbic I, Sanchez-Seco MP, Johnson N, Dottori M: Detection of mosquito-only flaviviruses in Europe. J Gen Virol 2012;93:1215-1225.

29 Evangelista J, Cruz C, Guevara C, Astete H, Carey C, Kochel TJ, Morrison AC, Williams M, Halsey ES, Forshey BM: Characterization of a novel flavivirus isolated from Culex ( $\mathrm{Me}$ lanoconion) ocossa mosquitoes from Iquitos, Peru. J Gen Virol 2013;94:1266-1272.

30 Crochu S, Cook S, Attoui H, Charrel RN, De Chesse R, Belhouchet M, Lemasson JJ, de Micco P, de Lamballerie X: Sequences of flavivirus-related RNA viruses persist in DNA form integrated in the genome of Aedes spp. mosquitoes. J Gen Virol 2004;85:1971-1980.
31 Vázquez A, Sánchez-Seco MP, Palacios G, Molero F, Reyes N: Novel flaviviruses detected in different species of mosquitoes in Spain. Vector Borne Zoonotic Dis 2012;12:223-229.

- 32 Ochieng C, Lutomiah J, Makio A, Koka H, Chepkorir E, Yalwala S, Mutisya J, Musila L, Khamadi S, Richardson J, Bast J, Schnabel D, Wurapa E, Sang R: Mosquito-borne arbovirus surveillance at selected sites in diverse ecological zones of Kenya; 2007-2012. Virol J 2013; 10:140.

33 Gonzalez-Reiche AS, Monzon-Pineda M de L, Johnson BW, Morales-Betoulle ME: Detection of West Nile viral RNA from field-collected mosquitoes in tropical regions by conventional and real time RT-PCR; in King $\mathrm{N}$ (ed): RT-PCR Protocol. New York, Humana Press, 2010, pp 109-124.

34 ICMR Bulletin (2000): Japanese encephalitis virus infection in mosquitoes and its epidemiological implications. http://icmr.nic.in/ buapr2000.pdf (accessed May 16, 2013).

- 35 Tewari SC, Thenmozhi V, Arunachalam N, Philip Samuel P, Tyagi BK: Desiccated vector mosquito used for the surveillance of Japanese encephalitis virus activity in endemic Southern India. Trop Med Int Health 2008; 13:286-290.

36 Borah J, Dutta P, Khan SA, Mahanta J: Epidemiological concordance of Japanese encephalitis virus infection among mosquito vectors, amplifying hosts and humans in India. Epidemiol Infect 2013;141:74-80.

37 Dutta P, Khan SA, Khan AM, Borah J, Chowdhury P, Mahanta J: First evidence of chikungunya virus infection in Assam, Northeast India. Trans R Soc Trop Med Hyg 2011;105: 355-357.

38 Khan SA, Dutta P, Khan AM, Chowdhury P, Borah J, Doloi P, Mahanta J: West Nile virus infection, Assam, India. Emerg Infect Dis 2011;17:947-948.

39 Sankari T, Hoti SL, Singh TB, Shanmugavel J: Outbreak of dengue virus serotype-2 (DENV2) of Cambodian origin in Manipur, India association with meteorological factors. Indian J Med Res 2012;136:649-655. 